\title{
Metodo y teoría en la evaluación de desayunos escolares
}

\author{
José Angel Vera Noriega \\ Centro de Investigación en Alimentación y Desarrollo A. C.
}

\section{Resumen}

Se llevó a cabo un estudio para evaluar el impacto de un programa de desayunos escolares en repertorios de discriminación, memoria y atención, comparando la zona urbana y rural. En la zona rural, fueron evaluados 300 niños elegidos al azar de los listados de preescolar que recibieron desayuno en los municipios en pobreza extrema y 150 , que no lo recibieron. En la zona urbana, fueron evaluados 300 niños seleccionados aleatoriamente de los listados de las escuelas ubicadas en las colonias marginadas que recibieron desayuno y 150 , que no recibieron desayuno.

En una prueba sintética de atención y memoria, los resultados muestran que en la zona urbana no existen diferencias en las medidas atribuibles al programa de desayunos. En la zona rural, los niños mejoraron en sus ejecuciones y tiempos. Se encontraron algunas diferencias relacionadas con el impacto del desayuno sobre niños con diferente estado nutricional. Los niños rurales se beneficiaron más que los urbanos del programa.

Palabras claves: desayunos escolares, comparación de medias, urbano e rural.

\section{Abstract}

Method and theory in school breakfast program evaluation In order to compare and evaluate a school breakfast program impact and discrimination memory and attention repertories on urban and rural children. A study was done 300 rural 
children were randomly chosed to have breakfast and 150 didn't have breakfast were evaluated and compared to 300 urban children who were randomly chose of extreme poverty marginal to have breakfast and 150 that didn't have breakfast. A designed test to evaluate attention and memory shows that in urban children do not exist differences between measures of the behavior. Rural children improve their time and performance. It was found differences on breakfast impact based on nutrimental status. The rural children got more benefits than the urban children with the program.

A pesar de que en México se están distribuyendo desayunos a los escolares desde hace mucho tiempo a través de diversos programas de ayuda social, son escasas las evaluaciones realizadas sobre el impacto de dichos programas y sus resultados. Aún no son muy claros en términos nutricionales, pues resulta difícil aislar o controlar el total de las variables relacionadas con su impacto sobre la salud o el desarrollo del niño.

Los programas de ayuda alimentaria pueden clasificarse en dos, según Rogers (1995): los de distribución directa, en los que los escolares reciben el desayuno y lo consumen dentro de la escuela y aquellos que consisten en la entrega de bonos a los beneficiarios, para que puedan cambiarlos por alimentos.

Aún cuando existe poca información sobre los beneficios logrados por los programas de apoyo de alimentos (Beaton \& Ghassemi, 1982), una conclusión de las evaluaciones existentes es que los programas son limitados en cuanto a su efecto sobre el estado nutricional y más bien apoyan la economía familiar. Sin embargo, su carácter humanitario y popular hace de estos programas una alternativa a la solución de problemas de alimentación en niños de zonas marginadas, mientras se instrumentan acciones macro y microeconómicas que resuelvan el problema (Sánchez-Griñan, Pérez, Jacoby, Yeager \& Barlett, 1992). 
Además de los objetivos nutricionales, los programas de desayunos escolares proponen objetivos educativos. Los niños desnutridos o con historia de desnutrición crónica disminuyen sus competencias cognitivas y su aprovechamiento en la escuela (Wilson, 1981). Por esto que los desayunos escolares se proponen mejorar el estado nutricional del niño y, al mismo tiempo, mejorar su desarrollo físico e intelectual, además de fomentar la asistencia del niño a la escuela, promover la socialización durante el desayuno en grupo y proveer la energía necesaria para la ejecución de conducta básica de atención y discriminación.

En los últimos años se han llevado a cabo diferentes estudios de evaluación sobre los efectos del ayuno en el funcionamiento intelectual de los niños (Pollitt, 1995; Chandler, Walker, Connolly \& GranthamMcGregor, 1995).

Estos estudios permiten, por un lado, observar la sensibilidad del sistema reactivo biológico a la falta de nutrientes y, por otro lado, revelan de qué manera los programas de merienda escolar impactan el proceso enseñanza - aprendizaje.

Existe un modelo biológico del hambre, derivado de un estudio de Pollitt, Leibel y Greenfield (1981) en situaciones controladas, que supone que se requiere una cantidad alta de glucosa en sangre al empezar el día, si no se quiere que al transcurrir del tiempo habilidades atentivas, discriminativas y de solución de problemas simples se vean menguadas.

Existen dos o más mecanismos a través de los cuales el suplemento alimentario incide en el rendimiento del niño en la escuela. El primero es a corto plazo y consiste en cambios metabólicos determinados por el mantenimiento de una fuente de energía que contribuya al funcionamiento cerebral. El período más largo en el día durante el cual el niño no dispone de un suministro de oxigeno de energía se da, por lo general, después de la cena y antes del desayuno. En el período entran en acción una serie de mecanismos autorreguladores, a fin de que los requerimientos energéticos del cerebro sean satisfechos siempre y el metabolismo cerebral funcione sin interrupción. Pero si el período de ayuno nocturno continua y se prolonga en la mañana, se 
producen algunos fenómenos, como, por ejemplo, la progresiva disminución de insulina y de glucosa asociada con otras modificaciones metabólicas que, a su vez, puede dar lugar a una situación de estrés orgánico, dificultando algunas de las funciones básicas del cerebro (como la fijación de la atención o la memoria a corto plazo). No se trata de modificaciones patológicas (las cuales pueden ocurrir si el ayuno se extiende por períodos más prolongados), sino de estados fisiológicos transitorios que incluyen alteraciones de en las capacidades cognitivas (Pollitt, Saco-Pollitt, Liebel \& Viterie, 1986).

Estos aspectos sirven para justificar que una suplementación a tiempo por la mañana podría optimizar algunos procesos psiquicos básicos en el niño. En este mismo estudio se informó que los niños con historia de desnutrición presentaban mayores problemas con sus habilidades cuando tenían hambre a media mañana.

Donald y McGregor (1989) examinaron los efectos de omitir el desayuno sobre las funciones cognoscitivas de tres grupos de niños de 9 a 10.5 años de edad, cada uno con 30 sujetos, los grupos estaban formados por un grupo de desnutridos, otro de niños sin desnutrición y un tercero con niños con desnutrición severa. Estos niños fueron admitidos veces a una guardería metabólica, después de no comer durante una noche; la mitad de los niños recibieron desayuno en su primera visita y una taza de té en su segunda visita. Ese orden de tratamiento fue invertida para la otra mitad. Las pruebas de funciones cognitivas que se utilizaron fueron las siguientes: el dígito Span test que involucra el recordar inmediatamente series de dígitos que incrementan en longitud; el test de codificación, donde los niños tienen que sustituir símbolos por números tan rápido como sea posible, el test de fluidez que mide generación de ideas y motivación y el test de comprensión atentiva que mide atención, memoria auditiva a corto plazo y trabajo de comprensión. Las otras pruebas utilizadas, el test de apareamiento de figuras familiares (MFFT) y el test de tarea centralincidental de Hagen (HCI), fueron previamente mostrados como sensibles a la omisión del desayuno. El primero mide habilidad de resolución de problemas y rapidez de respuesta y el segundo memoria visual a corto plazo, atención a las tareas e la información irrelevante. 
En los resultados, el grupo control no fue adversamente afectado en ninguno de los test cognitivos cuando el desayuno fue omitido. Aún tuvieron una mejor ejecución en aritmética y fueron mas eficientes en resolución de problemas. En contraste los grupos con desnutrición y desnutrición severa fueron afectados adversamente en fluidez (generación de ideas y motivación) y codificación (memoria visual a corto plazo). Los niños desnutridos fueron adversamente afectados en el test dígito span back wards. El recordar los dígitos hacia a atrás, es una medida de la memoria auditiva a corto plazo con un componente de procesamiento inmediato. Consecuentemente, los niños afectados por la pérdida del desayuno, como muestra en la mayoría de las pruebas cognitivas, fueron aquellos que estaban desnutridos. Ellos también fueron los únicos afectados en la eficiencia de la resolución de problemas. En conclusión, bajo condiciones controladas, los niños con desnutrición y desnutrición severa fueron adversamente afectados en algunas funciones cognitivas. Esto contrastado con los niños que no estaban desnutridos. Si los efectos aquí encontrados son replicados y se encuentra que continúan por un período de tiempo, entonces perder el desayuno puede contribuir fuertemente al logro escolar de los niños mal alimentados. La indicación es que donde los recursos son limitados, las comidas o desayunos escolares deberán dirigirse a niños mal nutridos que se verían más beneficiados que los niños adecuadamente nutridos en cuanto a los niveles de logro escolar.

Si bien es cierto que la evidencia apoya la asociación entre el estado nutricional y el desarrollo intelectual (Pollitt \& Thompson, 1997), también es cierto que diversas variables que se relacionan con el tipo de escuela a la que asiste el niño y las características de la comunidad, sea rural o urbana, pueden afectar el desarrollo intelectual. Por lo anterior, sería importante observar cómo el estado nutricional interactua con la variable rural-urbano con y sin desayuno para cada una de las pruebas de discriminación.

Tres preguntas resultan importantes: ¿Pueden variar los efectos del desayuno sobre el desarrollo cognoscitivo en función del estado nutricional del niño? ¿Existen diferencias en los efectos del desayuno según se trate de discriminación condicional, memoria a corto plazo o 
motora gruesa, es decir, en función del proceso psicológico que se evalúa? ¿El desayuno impactará de forma diferencial el comportamiento cognitivo de niños urbanos y rurales?

\section{Método}

\section{Sujetos}

Se estudian 450 niños de 5 a 6 años de edad en la zona rural y 450 niños de 6 a 7 años de edad en la zona urbana. Para esta investigación se eligieron municipios rurales y colonias urbanas en pobreza extrema según los criterios de Camberos, Genesta y Huesca (1994). Los listados de las escuelas ubicadas en los municipios rurales de Tesopaco, Quiriego, Alamos, Navojoa y Etchojoa y de aquellas ubicadas en las colonias Solidaridad III, 4 Olivos, Pedregal de la Villa, Villa Hermosa, Insurgentes y Progresista de Hermosillo y Sonora sirvieron como base muestral. Los niños seleccionados aleatoriamente de primer grado para la zona urbana y de tercer grado de preescolar para la zona rural, debían cumplir con las condiciones de estar inscritos en el año lectivo correspondiente, no ser repetidores y contar con la autorización de los padres para el estudio.

Una submuestra de 80 niños de la comunidad rural y 80 , de la urbana fueron pesados y medidos momentos antes de la evaluación psicológica. Todos estos niños recibieron desayuno en sus comunidades y su peso-talla servió para comparar el impacto del desayuno según el estado nutricional. Las medidas se tomaron antes y después del programa de desayunos que tuvo una duración de ocho meses.

Todos los niños consumieron su desayuno por la mañana antes de iniciar las clases y en ningún caso se permitió llevar el desayuno a la casa.

\section{Características del Desayuno}

Los menúes fueron formulados y balanceados de acuerdo a las recomendaciones internacionales para la distribución de la energía proveniente de proteína $(10-15 \%)$, grasa $(<30 \%)$ y carbohidratos $(55-$ $60 \%$ ). Con respecto al aporte de vitamina $A$, esta cumplió con el $30 \%$ 
de la recomendación para los escolares y $46 \%$ para preescolares. En cuanto al hierro los menúes proporcionaron del 57 al $89 \%$ de la recomendación. Es importante aclarar que aunque los menúes estuvieron equilibrados desde el punto de vista de energía, se recomendó la inclusión de alguna fruta de temporada, en especial la naranja ya que proporciona vitamina $C$, la cual es importante para la absorción del hierro. A los preescolares se les ofreció leche $(250 \mathrm{ml})$, cereal (30 gr) y galletas (30 gr) que variaban en su sabor o presentación en el tiempo (Grijalva-Haro, 1996).

\section{Medidas}

Los niños fueron evaluados antes y después del programa de desayunos, con un tiempo entre mediciones de ocho meses. Se aplicó una prueba de manera individual que ocupa entre 5 y 7 minutos.

Para medir cambios en la respuesta de selección por efecto de demoras interpuestas entre un estímulo selector y nueve de comparación, se utilizó el procedimiento de discriminación condicional (Cumming \& Berryman, 1976).

La memoria visual fue evaluada de dos formas: a través del tiempo de respuesta de una tarea de imitación de dos figuras trazadas en el aire por el evaluador (caracol y m patona) y por medio de la imitación textual de los dos estímulos. En la reproducción textual se midió el tiempo transcurrido desde que el niño colocaba el lápiz sobre el papel hasta que terminaba el trazo. La adecuación del trazo (correcto e incorrecto) se evaluó según su correspondencia con el estímulo muestra. Para memoria auditiva siete palabras fueron escuchadas por el niño (silla, mesa, piedra, árbol, casa, carretera, flor) contando las recordadas y las repetidas sin problemas de pronunciación. Para conducta verbal, sólo se atendió a la pronunciación, para esto, se utilizaron 10 palabras complejas, como cosmopolitanismo, sardanápalo, ingrediente, etc. contando el total de palabras bien pronunciadas. Para evaluar la sincronía de los movimientos motores gruesos se registró el tiempo de ejecución de una carrera partiendo de la posición de pie y una segunda carrera partiendo de posición supina en una distancia de 15 metros. 
En el caso particular de las habilidades cognitivo-perceptuales se requiere no sólo juzgar su presencia o ausencia, sino determinar el nivel de dominio en tareas de complejidad creciente. Con base en estas consideraciones se estructuró un instrumento que consta de siete subescalas: fluidez verbal, ordenación de dibujos, figuras incompletas, logro visual, diseño con prismas, retención de dígitos y laberintos. Dichas subescalas fueron aplicadas en el orden enunciado, alternando los tipos de prueba para disminuir la fatiga y mantener el interés del niño. La estructuración de los reactivos de prueba se hizo para niños de 6 a 7 años de edad.

Los niños fueron evaluados de manera individual en sus escuelas, fuera de su aula de clase y bajo condiciones improvisadas, pero tecnicamente confiables.

\section{Resultados}

\section{Evaluación Sintética de Atención y Memoria}

Con el objeto de reducir el número de variables se llevó a cabo un análisis factorial de componentes principales y rotación varimax (SPSS, 1997) los resultados presentaron tres componentes para los tiempos con una varianza explicada de 58.4\% y cargas factoriales mayores a .50. Se agruparon en el primer componente la carrera supina y de pie, en el segundo, tiempo textual caracol y m patona y las latencias de respuesta para la tarea de elección se agruparon en el tercer componente. Para las ejecuciones tenemos tres factores con una varianza explicada de $73.36 \%$ y cargas factoriales mayores a .80. En el primer factor se agruparon palabras recordadas y pronunciación de palabras complejas, en el segundo reproducción textual y aérea de la M; tercero, reproducción textual y aérea del caracol y cuarto los valores de ejecución para las respuestas de elección.

Como puede observarse no existen diferencias en los cambios observados en uno y otro grupo.

En el grupo urbano, la latencia en la respuesta de elección paso de 6.87 segundos a 5.47, implicando un cambio más significativo que el que se observa en el grupo sin desayuno. El puntaje de acierto fue de 
Tabla 1. Resultados del constraste t de student para grupos con y sin desayunos antes y después del programa en la zona urbana.

\begin{tabular}{lrrrrrrrr}
\hline & \multicolumn{3}{c}{ Con desayuno $(\mathrm{n}=280)$} & \multicolumn{4}{c}{ Sin desayuno $(\mathrm{n}=143)$} \\
& Antes & Después & \multicolumn{1}{c}{$\mathrm{T}$} & \multicolumn{1}{c}{$\mathrm{p}$} & Antes & Después & \multicolumn{1}{c}{$\mathrm{t}$} & $\mathrm{p}$ \\
\hline Latencia elección & 6.87 & 5.47 & 4.33 & $\mathbf{. 0 0 0}$ & 6.56 & 5.27 & 3.15 & $\mathbf{. 0 0 2}$ \\
Puntaje elección & 2.55 & 2.63 & 1.61 & .107 & 2.47 & 2.67 & 2.79 & $\mathbf{. 0 0 6}$ \\
Tiempo reproducción & 9.30 & 9.29 & .007 & .99 & 9.58 & 10.37 & 1.24 & .217 \\
Puntaje caracol & 1.01 & 1.03 & .463 & .644 & .96 & 1.05 & 1.19 & .235 \\
Puntaje M & 1.58 & 1.75 & 3.15 & $\mathbf{. 0 0 2}$ & 1.62 & 1.71 & 1.22 & .223 \\
Recordadas pronunciadas & 7.83 & 9.06 & 7.56 & $\mathbf{. 0 0 0}$ & 7.88 & 9.20 & 5.80 & $\mathbf{. 0 0 0}$ \\
Carrera & 13.00 & 12.87 & .36 & .173 & 12.98 & 12.81 & 1.41 & .159 \\
\hline
\end{tabular}

2.55 a 2.63 de un máximo de 3 en el grupo con desayuno. Para este caso, el grupo sin desayunos inició con mayor número de errores (.53) que el grupo con desayunos (.45), sin embargo, el nivel de avance fue de .08 y .20 para los grupos con y sin desayuno respectivamente (Tabla 1).

Para el grupo sin programa o de comparación, en la zona rural, las velocidades de respuesta mejoraron significativamente en la carrera de pie y supina, tareas de recuerdo y repetición (Tabla 2).

Tabla 2. Resultados del contraste t de student para grupos con y sin desayuno antes y después del programa en la zona rural.

\begin{tabular}{lrrrrrrrr}
\hline & \multicolumn{3}{c}{ Con desayuno $(\mathrm{n}=280)$} & \multicolumn{4}{c}{ Sin desayuno $(\mathrm{n}=143)$} \\
\hline & Antes & Después & \multicolumn{1}{c}{$\mathrm{T}$} & $\mathrm{p}$ & Antes & Después & $\mathrm{t}$ & $\mathrm{p}$ \\
\hline Latencia elección & 8.72 & 7.98 & 1.56 & .119 & 7.83 & 7.42 & .668 & .505 \\
Puntaje elección & 2.03 & 2.27 & 3.87 & $\mathbf{. 0 0 0}$ & 2.27 & 2.57 & 3.67 & $\mathbf{. 0 0 0}$ \\
Tiempo reproducción & 15.57 & 10.00 & 5.26 & $\mathbf{. 0 0 0}$ & 11.78 & 11.13 & .751 & .454 \\
Puntaje caracol & .59 & 1.35 & 12.9 & $\mathbf{. 0 0 0}$ & .55 & 1.27 & 9.67 & $\mathbf{. 0 0 0}$ \\
Puntaje M & 1.00 & 1.36 & 5.48 & $\mathbf{. 0 0 0}$ & 1.20 & 1.55 & 4.52 & $\mathbf{. 0 0 0}$ \\
Recordadas pronunciadas & 6.95 & 7.74 & 4.57 & $\mathbf{. 0 0 0}$ & 7.30 & 8.48 & 5.87 & $\mathbf{. 0 0 0}$ \\
Carrera & 12.03 & 10.89 & 8.3 & $\mathbf{. 0 0 0}$ & 11.45 & 11.24 & 1.72 & .087 \\
\hline
\end{tabular}

Al compararlos, la ejecución final del grupo que recibió el programa presenta en su post prueba velocidades de respuesta en tareas de cognición que son muy parecidas a la pre prueba del grupo sin programa. Una comparación t de student entre la post prueba del grupo con programa y la pre prueba del grupo sin programa, indica que no 
existe alguna diferencia estadísticamente significativa entre ambas puntuaciones $(\mathrm{t}=.68 ; \mathrm{p}=.63 ; \mathrm{gl}=153)$, esto es, el punto de partida del grupo sin programa es casi el mismo que el punto de llegada del grupo con programa; aún cuando ambos han mejorado.

Una vez clasificados los niños en base a su peso para la talla se obtuvieron las medias y pruebas de contraste de hipótesis sólo para los niños con desayuno de la zona urbana y rural en cada uno de los reactivos aplicados en la post prueba (Tabla 3 ).

Tabla 3. Resultado del contraste t de student para el grupo rural y urbano con desayuno por abajo y por arriba de la primera desviación estándar del peso para la talla.

\begin{tabular}{lccrc}
\hline & \multicolumn{4}{c}{ Niños urbanos y rurales con programa } \\
\cline { 2 - 5 } & Grupo $1(-1<)$ & Peso/talla & Grupo $2(>+1)$ & \multicolumn{1}{c}{$\mathrm{p}$} \\
\hline Tiempo de elección & 9.60 & 7.23 & 2.300 & .02 \\
Tiempo de reproducción & 11.83 & 11.59 & .177 & .86 \\
Reproducción & 2.07 & 2.54 & 1.720 & .08 \\
Palabra & 7.46 & 7.91 & .795 & .43 \\
Elección & 2.15 & 2.17 & .094 & .92 \\
Carrera & 11.39 & 10.75 & 1.720 & .08 \\
$\mathrm{n}$ & 65 & 34 & & \\
\hline
\end{tabular}

Curiosamente se observa que el tiempo de elección no resultó diferente por efectos del desayuno si es importante en niños con y sin problemas nutricionales. Se observa en la tabla 3, que los niños con menor peso para la talla obtienen tiempos significativamente mayores. El reactivo de reproducción textual y aérea del caracol y la M presentan una media de 2.07 en niños por debajo de la -1 desviación estándar (D.S.) y 2.54 para los niños por arriba de la +1 D.S. En la velocidad de carrera, los niños mejor nutridos presentaron tiempos más cortos de llegada a la meta.

La Tabla 4 es sumamente ilustrativa de los apoyos al desarrollo que el programa de desayunos escolares puede brindar fundamentalmente a la zona rural. Todas las diferencias significativas favorecen al niño de la zona rural que presenta en la tabla 1 y 2 de 280 niños con 
Tabla 4. Resultados del contraste t de student para el grupo con desayuno después del programa comparando zona urbana y rural.

\begin{tabular}{lrrrc}
\hline & Urbana & Rural & $\mathrm{t}$ & $\mathrm{p}$ \\
\hline Tiempo de elección & 9.74 & 8.52 & .90 & .36 \\
Tiempo de reproducción & 13.57 & 9.46 & 3.83 & $\mathbf{. 0 0}$ \\
Reproducción & 1.64 & 2.90 & 5.49 & $\mathbf{. 0 0}$ \\
Palabra & 7.03 & 8.29 & 2.56 & $\mathbf{. 0 1}$ \\
Elección & 1.92 & 2.49 & 3.43 & $\mathbf{. 0 0}$ \\
Carrera & 11.66 & 10.63 & 2.87 & $\mathbf{. 0 0}$ \\
$\mathrm{n}$ & 55 & 49 & & \\
\hline
\end{tabular}

desayuno, los niveles de entrada más bajos en todos los reactivos. Como se observa, los tiempos de elección, reproducción y carrera son más cortos para el grupo de la zona rural y las ejecuciones son mejores en número de palabras recordadas y repetidas, reproducción de caracol y M y en el puntaje elección.

\section{Evaluación Extensa de Atención-Memoria y Cognición}

Con el objeto de reducir el número de variables se llevó a cabo un análisis factorial de componentes principales con rotación varimax (SPSS, 1997). Los resultados presentaron tres componentes con una varianza explicada de $48.9 \%$ y cargas factoriales mayores a .50. El primer factor agrupó los tiempos en la solución de laberintos, el segundo agrupó tiempos en el diseño de prismas y el tercero la secuencia de tarjetas. Para el análisis factorial de los datos de puntaje se obtuvieron tres componentes con pesos factoriales mayores a .50. El primer factor agrupó los puntajes en el diseño de prismas, el segundo la solución de laberintos y el tercero la secuencia de tarjetas.

Comparando los niveles de entrada para los grupos con y sin desayuno no se encontró diferencia significativa en las medidas de tiempo y ejecución para los reactivos de prueba.

Observando las medias y los resultados del contraste $t$ student antes y después podríamos suponer que el consumo de desayunos mejora significativamente el tiempo utilizado en el diseño de prismas, pero además se relaciona con un decremento en los tiempos para la 
Tabla 5. Medias y pruebas $t$ significativas para la comparación antes y después del grupo con desayunos y sin desayunos.

\begin{tabular}{lcccc}
\hline & \multicolumn{2}{c}{ Con Desayuno } & \multicolumn{2}{c}{ Sin Desayuno } \\
\cline { 2 - 5 } & Antes & Después & Antes & Después \\
\hline Tiempo diseño & 101.9 & $82.2^{* * *}$ & 99.8 & 99.7 \\
Tiempo laberinto & 47.7 & $32.9^{* * *}$ & 38.1 & $30.5^{* *}$ \\
Tiempo secuencia & 44.4 & $37.2^{* * *}$ & 46.0 & $33.5^{* *}$ \\
Puntaje diseño & 7.5 & $8.6^{* * *}$ & 7.6 & $9.0^{* *}$ \\
Puntaje laberinto & 7.4 & $8.4^{* * *}$ & 7.9 & $8.5^{* * *}$ \\
Puntaje secuencia & 3.3 & $4.6^{* *}$ & 3.4 & $4.6^{* * *}$ \\
Logro visual & 52.3 & $61.2^{* * *}$ & 51.4 & $59.3^{* *}$ \\
Productos & 23.1 & $27.1^{* *}$ & 24.3 & $25.7^{*}$ \\
\hline$* * *<001 \cdot * * \mathrm{p}<01 * *_{\mathrm{p}}<05$ & & &
\end{tabular}

solución de laberintos que inicia con un valor de 47.7 y termina con 32.9 segundos y tiempos de secuencias que va de 44 a 37 segundos. Aún cuando en estos dos últimos casos ambos contrastes son significativos la diferencia es mayor en el grupo con desayunos por lo que sus valores de significancia son más altos.

\section{Discusión}

En síntesis y con relación a los efectos globales del programa para niños en la zona rural, se puede anotar que los niños que recibieron desayuno mejoraron sus tiempos de reacción y sus ejecuciones en tareas cognoscitivas y verbales de manera estadísticamente significativa. Sin embargo, la comparación del grupo con y sin desayunos al final del programa no presenta diferencias significativas. Debe notarse que el grupo control ilustra el efecto de la variable maduración durante el tiempo del programa, por lo que se podría decir que el impacto fundamental del programa de desayunos fue equilibrar el proceso de desarrollo del niño, dando a los niños con programa que viven en condiciones de desaventaja el mismo nivel de posibilidades cognitivas y verbales que a un niño con mejores condiciones de estimulación.

Según los datos reportados por Grijalva-Haro (1996), los niños de la zona rural dentro del programa, el porcentaje de riesgo leve (entre $1 \mathrm{y}-2$ ) paso de $14.8 \%$ al inicio y $10.9 \%$ al final de seis meses y en el 
grupo sin programa, de $9.9 \%$ a $12.2 \%$, es decir, aumentó un $2.3 \%$. Los niños por abajo del corte de $-2 \mathrm{z}$ para peso/talla fue menor al $1 \%$ al inicio y al final. Por lo anterior, los niños ubicados debajo del corte de -1 y -2 presentan un peso que se encuentra más cerca de la menos una desviación estandar.

Si bien es cierto que los niños de cada uno de los grupos de comparación de la zona rural fueron elegidos al azar, las condiciones previas de elección al programa de desayunos les imponen diferencias pues siempre los niños sin programa se encontraban en escuelas y barrios en mejores condiciones. Los niños seleccionados en la zona urbana son aquellos con mayor número de carencias y privaciones con un contexto familiar pobre y conflictivo. En la zona urbana fue posible seleccionar de la misma aula a los niños de ambas condiciones controlando la variable relacionada con el maestro y el proceso de enseñanza-aprendizaje, sin embargo no pudo evitarse que los niños no seleccionados tuvieran alguna desventaja social y familiar que puede impactar sus posibilidades atentivas o cognoscitivas. En la zona rural, los niños del grupo sin desayunos fueron seleccionados de escuelas pertenecientes a un Municipio que decidió no canalizar recursos al programa. En este caso la diferencia entre los niños en los grupos es mayor debido a las condiciones de las escuelas, la permanencia y dedicación de los maestros fue diferente.

Por otro lado, la evidencia presentada sobre el impacto de los desayunos en velocidades de respuesta y ejecución de las tareas de imitación y discriminación compleja no necesariamente implica cambios en el desempeño y rendimiento en la escuela. Aún cuando estos repertorios básicos son precurrentes de conducta más compleja, el rendimiento no fue evaluado.

Las diferencias encontradas en niños por debajo de la menos una desviación estándar y los que se encuentran por arriba indican que el estado nutricional es un factor importante que restringe en el niño sus posibilidades de desarrollo. Los niños con menor peso para la talla se benefician en sus velocidades de elección y reproducción.

Si recordamos que los niños de la zona urbana durante el estudio cursaron el primer año de primaria (6-7) y los de la zona rural el tercer 
grado de preescolar (5-6) las diferencias en los puntos ganados después del programa de desayuno son muy alentadores.

Para la zona urbana y en términos de los resultados descritos se podría resumir que el programa de desayunos decrementa los tiempos de respuesta en tareas complejas de diseño de figuras lo mismo que en la solución de laberintos. Es posible que las tareas de elección, vocabulario y memoria visual de la prueba sintética se modifiquen en el transcurso de tiempo por efecto de entrenamiento en la escuela oscureciendo el efecto del programa. Estas tareas constituyen comportamientos muy básicos precurrentes para la lectura y la escritura, por las cuales, reciben atención en la escuela, lo que puede maximizar su ejecución en los niños. La carrera de pie y supina que encontramos dentro de la dimensión carrera no presenta diferencias significativas. La falta de nutrientes, relacionado con el ayuno, afecta de manera más severa los repertorios atentivos y discriminativos que los de tipo motor.

Los datos obtenidos en este estudio, comparados con el estudio de Perú (Pollitt, Jacoby \& Cueto, 1996) y Jamaica (Chandler et al., 1995) hacen suponer que los desayunos entregados de manera sistemática durante el ciclo escolar mejoran las ejecuciones en discriminación, memoria y tareas cognitivas básicas, tanto en niños de 5 a 7 como de 9 a 11 años. Sin embargo, los repertorios verbales no parecen verse afectados en niños de 5 a 7 años, mientras que existe evidencia en cambios de vocabulario en niños de 9 a 11 años.

Por los resultados obtenidos, se puede afirmar que el desayuno mejora las velocidades de respuesta en operaciones cognitivas que requieren de atención y discriminación y mejora la ejecución. Sin embargo, los adelantos en procesos básicos no podrán impactar el rendimiento académico, si esto no se ve reforzado con un programa de educación a padres y capacitación de maestros. Se recomienda que el programa incluya criterios claros y socialmente válidos de inclusión, como el peso para la talla, la frecuencia de las enfermedades respiratorias y diarreicas y/o las condiciones de riesgo socioeconómicas. Primero, porque se pierden recursos en los niños que no necesitan desayuno; segundo, porque el desayuno en niños con sobrepeso puede provo- 
car obesidad y tercero, porque si el programa sigue una política populista dejará de lado el impacto que pretende sobre el desarrollo del niño en la escuela.

Dirigiendo el programa de esta manera, la mitad de los recursos se economizan y con esta parte podrían complementarse los factores relacionados con el desarrollo educativo.

Vale la pena mencionar que actualmente se están haciendo esfuerzos para que los Municipios retomen el programa y con sus recursos y, asesoría profesional, diseñen dietas y, con lo disponible de la industria local, se elaboren menúes con el fin de que la comunidad se vea beneficiada y a su vez perciba como se benefician los niños.

\section{Referencias}

Beaton, G. H., \& Ghassemi H. (1982). Suplementary feeding programs for young children in developing countries. American Journal of Clinical Nutrition, 35, 267-276.

Camberos, M., Genesta, M. A., \& Huesca, L. (1994). La pobreza en Sonora: los límites a la modernización. Revista de Estudios Sociales, 15 (9), 167-197.

Chandler, A. M., Walker, S. P., Connolly, K., \& Grantham-McGregor, S. M. (1995). School breakfast improves verbal fluency in undernorished Jamaican children. Community and International Nutrition, 125(4), 894-899.

Cumming, W., \& Berryman, R. (1976) Algunos datos sobre la conducta de igualación del pichón. In C. Catania (Org.). Investigación Contemporánea en Conducta Operante. (pp. 218-221). México: Trillas.

Donald, T. Y., \& McGregor, G. (1989). Effects of missing breakfast on the cognitive functions of school children of differing nutritional status. American Journal of Clinical Nutrition, 49, 646-656.

Grijalva-Haro, M. I. (1996). Evaluación del impacto del programa de desayunos escolares (Proyecto de Investigación). Hermosillo: Secretaría de Educación y Cultura. Centro de Investigación en Alimentación y Desarrollo A. C.

Pollitt, E. (1995). Does breakfast make a difference in school? Journal of the American Dietetic Association. 95 (10), 1134-1139.

Pollitt, E., Jacoby, E., \& Cueto, S. (1996). School breakfast and cognition among nutritionally at risk children in the Peruvian Andes. Nutrition Review, 54 (4), 22-26.

Pollitt, E., Leibel, R.L., \& Greenfield, D. (1981). Brief fasting, stress and cognition in children. American Journal of Clinical Nutrition, 34, 1526-1533 
Pollitt, E., Saco-Pollitt, R.F., Leibel, R.L., \& Viterie, E. (1986). Iron deficiency and behavioral development in infants and preschool children. American Journal of Clinical Nutrition, 43, 555-565.

Pollitt, E., \& Thompson, C. (1997). Protein-calorie malnutrition and behavior: a view point from psychology. In R. G. Wurtman, \& J. J. Wurtman (Orgs.). Nutrition and the Brain. (vol. 2, pp. 10-57). New York: Raven Press.

Rogers, B. L. (1995). Feeding programs and food-related income transfers. In P. Pinstrup-Andersen, D. Pelletier, \& H. Alderman (Orgs.), Child growth and nutrition in developing countries: priorities for action (pp. 199-219). Ithaca: Cornell University Press.

Sánchez-Griñan, M., Pérez F., Jacoby E., Yeager, B., \& Bartlett, J. (1992). Las investigaciones e intervenciones nutricionales en el Perú: un análisis preliminar. Reporte Final (USAID Sub-contract: AID-PIO-T No. 52700003-10076) no publicado.

SPSS (1997). Estadísticas avanzadas. Chicago: Illinois, SPSS.

Wilson, A. (1981). Longitudinal analysis of diet, physical growth, verbal development, and school performance. In J. Balderson, A. Wilson, M. Freire \& M. Simonen (Orgs.). Malnourished children of the rural poor (pp. 39-81). Boston: Auburn House.

José Angel Vera Noriega, doutor em Psicologia Social pela Universidade Nacional do México, é pesquisador do Centro de Investigación en Alimentación y Desarrollo A. C.,

Departamento de Desarrollo Humano y Bienestar Social. Endereço para correpondência: Carretera a la Victoria Km. 0.6 Apdo. Postal 1735, Hermosillo, Sonora, México. Telefones: ++52(62)80-00-49 у 80-02-93; Fax. ++52(62) 80-00-55 y 80-04-85. E-mail. Avera@cascabel.ciad.mx. 